\title{
Toward Active-Site Compression in Rigid-Rod $\beta$-Barrels
}

\author{
Yoann Baudry*, Dawn Ronan, and Stefan Matile
}

\begin{abstract}
Applying the Woodhull equation to organic chemistry, a concept to maximize remote control of chemical processes that take place within synthetic multifunctional pores is described.
\end{abstract}

Keywords: $\beta$-Barrels · Bioorganic chemistry · Enzyme mimics · Ion channels · Molecular recognition

The rational design of $p$-octiphenyl $\mathbf{1}$ (Fig. 1) is based on the report in 1973 by A. M. Woodhull that the "ionic blockage of sodium channels in nerves" can be described with the following Eqn.:

$$
\begin{aligned}
\log K_{\mathrm{D}}= & \log K_{\mathrm{D}}(V=0 \mathrm{mV}) \\
& -\left(l_{\mathrm{A}} z F V\right) /(2.303 l R T)
\end{aligned}
$$

This Eqn., today referred to as the Woodhull equation, defines the dependence of the dissociation constant $\left(K_{\mathrm{D}}\right)$ of the complex formed by a neuronal ion channel and its blocker on the polarization of the lipid bilayer membrane [1]. Besides the usual constants with the usual meanings ( $F$, $R$ and in most cases also temperature $T$ ), voltage dependence of this biological example of molecular recognition decreases with the length $l$ of the channel and increases with the charge $z$ of the blocker. The most interesting parameter, however, is the 'Woodhull distance' $l_{\mathrm{A}}$, the distance from channel entrance to the active site. For a neurophysiologist, the Woodhull equation, therefore, can be used to determine the location of the active site in an ion channel or a pore. For an organic chemist, the Woodhull equation may become useful to maximize the remote control of chemical

${ }^{\star}$ Correspondence: Y. Baudry

Department of Organic Chemistry

University of Geneva

$\mathrm{CH}-1211$ Geneva

Tel.: +41223796514

Fax: +4122379 3215

E-Mail: yoann.baudry@chiorg.unige.ch processes that take place in synthetic multifunctional pores by rational design: the options are to increase $z, l_{\mathrm{A}}$ or both.

For us, the Woodhull equation has served well in studies on molecular recognition and catalysis that takes place within the confined and oriented nanospace of synthetic multifunctional pores [2]. In these pores, rigid-rod $p$-octiphenyl 'staves' are introduced to preorganize the cylindrical self-assembly of artificial $\beta$-barrels, where- as the $\beta$-sheet 'hoops' allow for straightforward internal and external pore design. In synthetic multifunctional pore 2 , for example, hydrophobic leucine residues are positioned at the exterior because of their sufficient bilayer affinity and $\beta$-propensity (Fig. 2) [3]. The interior of this pore is covered with histidine residues to interact with molecules passing by. However, pore 2 is not a convincing supramolecular host of organic anions like pyrene-1,3,6-trisulfonates (HPTS: $K_{\mathrm{D}}=1.5 \mathrm{mM}$ ). It is similarly un-

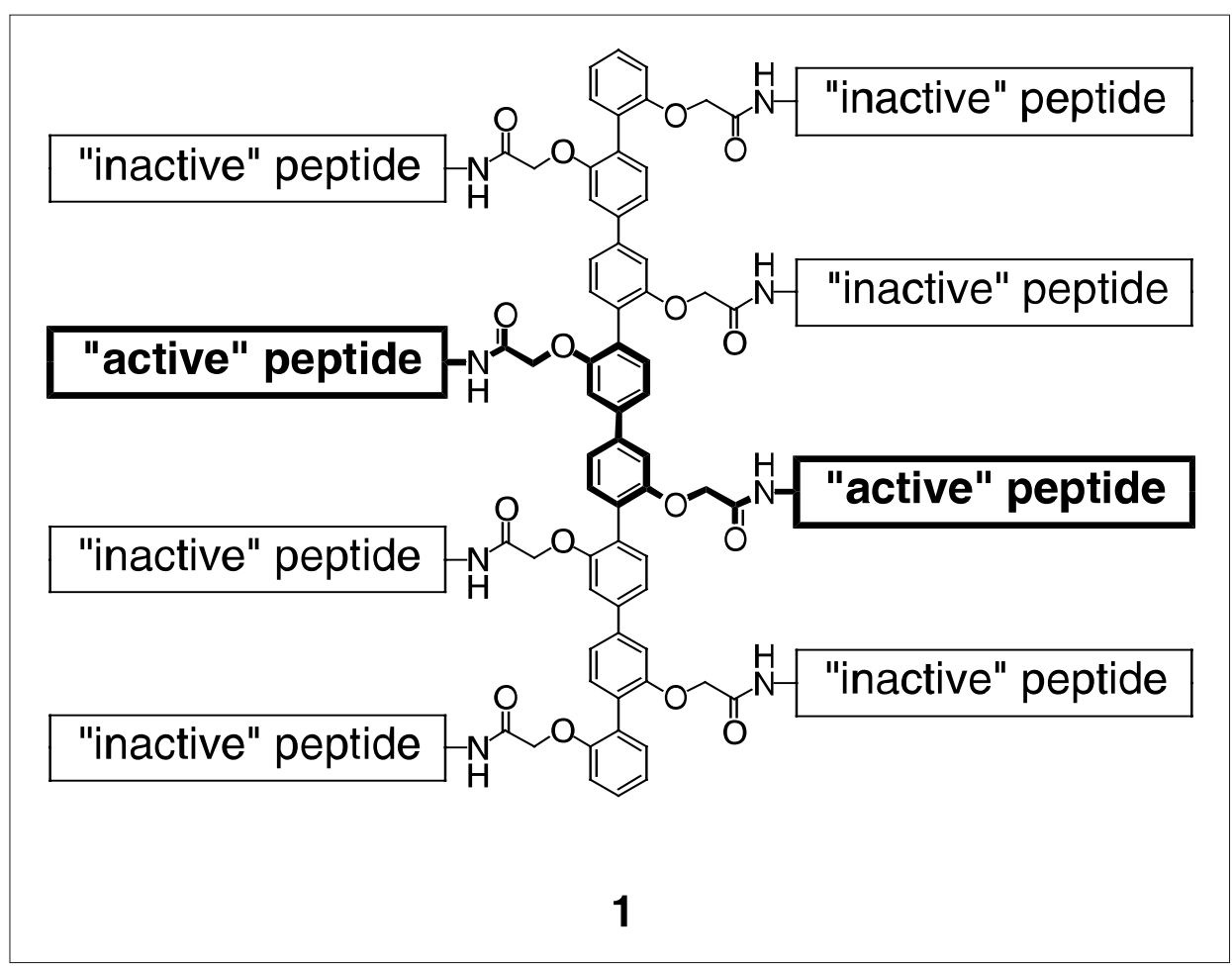

Fig. 1. According to the Woodhull equation, self-assembly of $p$-octiphenyl $\mathbf{1}$ into a $\beta$-barrel with internal active sites only in the middle should secure maximal remote control of chemical processes that take place in the resulting synthetic multifunctional pore. 


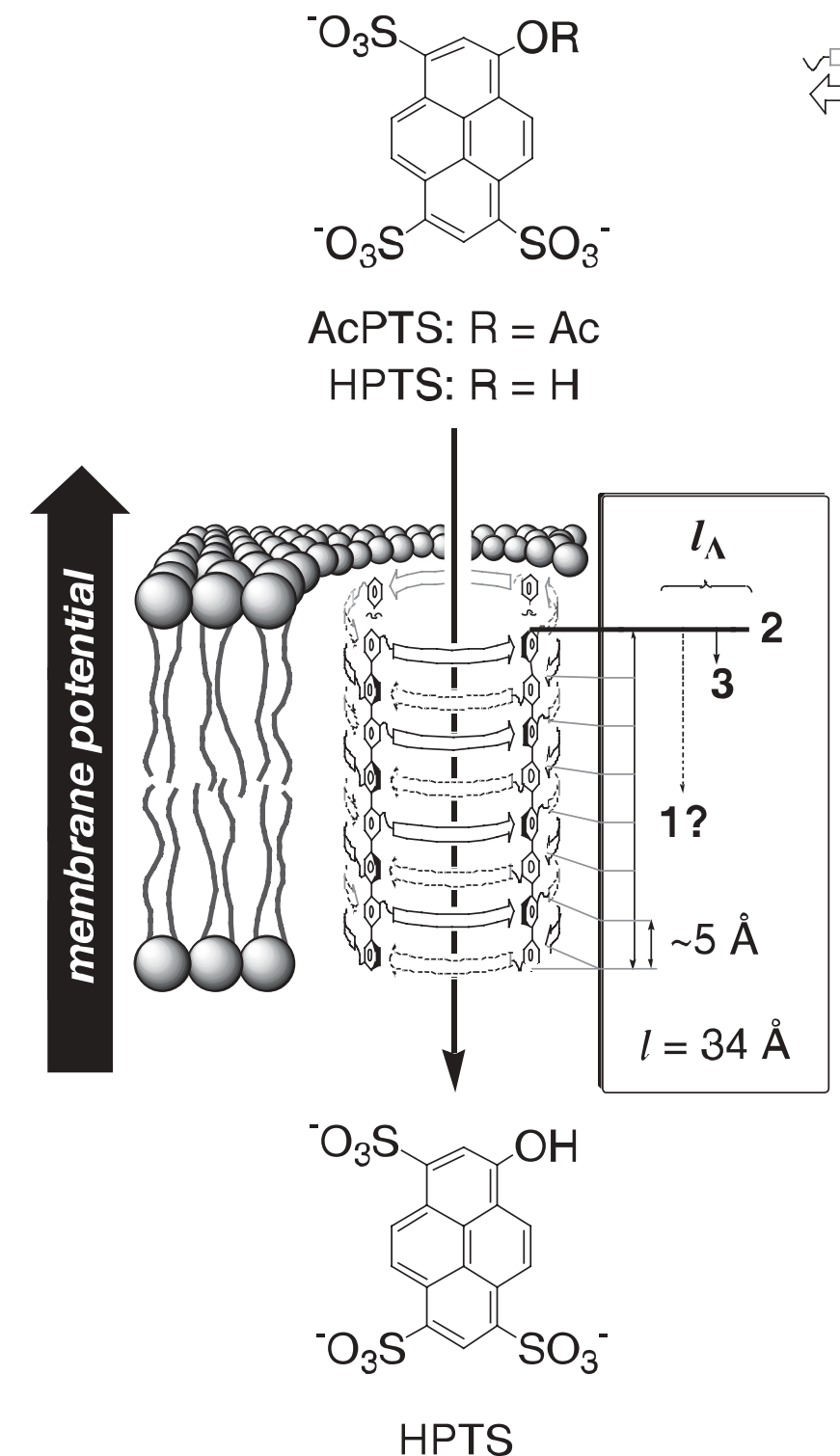

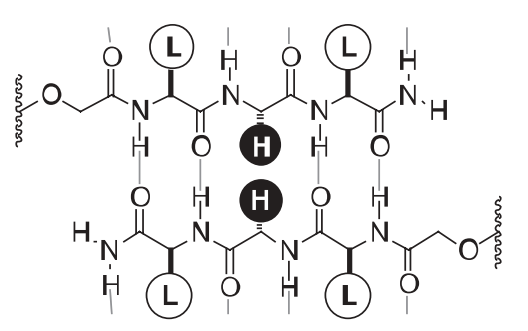

2: $l_{\mathrm{A}}=0.9 \AA$, sequence $=\mathrm{LHL}$

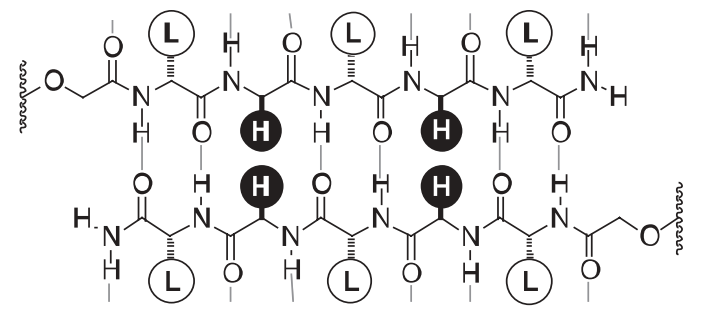

3: $l_{\mathrm{A}}=2.7 \AA$, sequence $=\mathrm{LHLHL}$

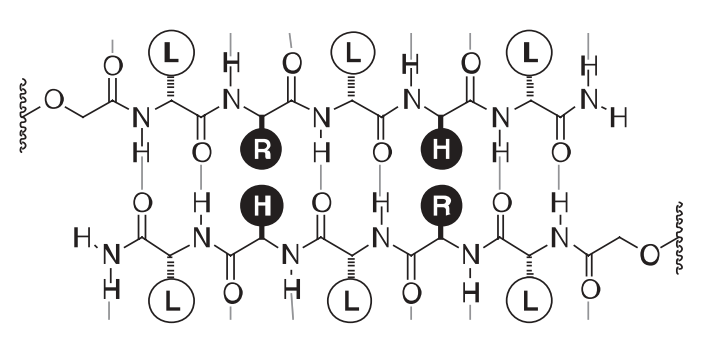

4: sequence $=\mathrm{LRLHL}$

Fig. 2. Woodhull distances $I_{\mathrm{A}}$ of rigid-rod $\beta$-barrel pores 2 and $\mathbf{3}$ and experimental setup for remote control of molecular recognition (e.g. HPTS, $\alpha$-helices) and catalysis (e.g. AcPTS $\rightarrow$ HPTS) within pores $\mathbf{3}$ and $\mathbf{4}$ by membrane polarization. External amino-acid residues (singleletter abbreviations) are depicted black on white, internal ones white on black. See review [2] and original literature for details.

able to catalyze the hydrolysis of esters like AcPTS.

Woodhull analysis provided a meaningful explanation for the poor performance of pore $2: l_{\mathrm{A}}=0.9 \AA$. This presumably negligible Woodhull distance suggests that neither the organic guest nor the substrates enter the pore. Planar bilayer conductance experiments confirmed that the inner diameter of pore $2(d \approx 5.2 \AA)$ is insufficient to include guests of the size of HPTS.

The situation improves dramatically upon pore expansion by $\beta$-sheet elongation. The interior of pore $\mathbf{3}$ is sufficient $(d \approx 7.0$ $\AA)$ to include $\left(K_{\mathrm{M}}=0.7 \mu \mathrm{M}\right)$ and hydrolyze AcPTS in a more respectable manner $\left[\left(k_{\text {cat }} / K_{\mathrm{M}}\right) / k_{\text {uncat }}=7.1 \times 10^{9} \mathrm{M}^{-1}\right]$ [4][5]. Mutation of every other histidine into arginine gives the expected improvement in molecular recognition and catalysis by pore 4 with internal arginine-histidine dyads [5][6]. Here, the situation seems set up to 'do' organic chemistry within synthetic pores. As expected from the Woodhull equation, membrane polarization can indeed be used to attract $\alpha$-helices into pore 4 [6]. With the same pore, electrostatic steering by supportive voltage was explored as attractive method to guide substrate AcPTS into and product HPTS out of the active sites of pore 4 to maximize catalysis [5].

According to the Woodhull equation, there is much room to improve upon these initial results. Compared to contracted pore 3, the Woodhull distance in expanded pore 4 increases by a factor of three [4]. Compared to a more than $1^{\prime} 000$-fold increase in molecular recognition, this result may surprise. However, $l_{\mathrm{A}}=2.7 \AA$ corresponds to about half of the distance between two strands in a $\beta$-sheet. The rate-limiting association of HPTS guests detected by Woodhull analysis occurs, therefore, with the first available internal histidines in the two peripheral strands of pore hosts $\mathbf{3}$. Subsequent guest 'hopping' through the pore and final exit into the media may then require less activation energy [7]. 
According to the Woodhull equation, detectable steering effects for both molecular recognition as well as catalysis using synthetic multifunctional pores with $l_{\mathrm{A}} \approx$ $2.7 \AA$ are extremely promising. Imagine the situation with $l_{\mathrm{A}} \approx 17 \AA$ ! To realize this situation, $p$-octiphenyl 1 was designed. In $p$ octiphenyl 1, peptide strands with 'active' sequences like LRLHL are attached only to the two central arenes. The six peripheral arenes are equipped with 'inactive' arginine- and histidine-free peptides. Self-assembly of $p$-octiphenyl 1 into a $\beta$-barrel should then produce a pore with active sites only in the middle. Synthetic efforts toward p-octiphenyl $\mathbf{1}$ and related rods are in progress and will be reported in due course [8].

Financial support of this research by the Swiss NSF is gratefully acknowledged (200020-101486 and National Research Program "Supramolecular Functional Materials" 4047-057496).

Received: January 19, 2004

[1] A.M. Woodhull, J. Gen. Physiol. 1973, 61, 687-708.

[2] N. Sakai, S. Matile, Chem. Commun. 2003, 2514-2523.
[3] A. Som, N. Sakai, S. Matile, Bioorg. Med. Chem. 2003, 11, 1363-1369.

[4] B. Baumeister, N. Sakai, S. Matile, Org. Lett. 2001, 3, 4229-4232.

[5] N. Sakai, N. Sordé, S. Matile, J. Am. Chem. Soc. 2003, 125, 7776-7777.

[6] N. Sordé, S. Matile, J. Supramol. Chem. 2002, 2, 191-199.

[7] D. Ronan, N. Sordé, S. Matile, submitted.

[8] D. Ronan, Y. Baudry, S. Matile, unpublished results.

\title{
Development of a Robust Capillary Electrophoresis-Mass Spectrometer Inter- face with a Floating Sheath Liquid Feed
}

\author{
Pascal Bernet ${ }^{a \star}$, Dominik Blaser ${ }^{a}$, Stefan Berger ${ }^{\mathrm{b}}$ and Martin Schär ${ }^{\mathrm{a}}$
}

\begin{abstract}
The on-line combination of capillary electrophoresis (CE) with electrospray ionisation mass spectrometry (ESI-MS) has attracted major attention for the in-depth analysis of complex samples. However, CE-MS coupling is not straightforward. We present a novel coaxial sheath liquid CE-MS interface, which is robust and can be used for both hyphenated techniques, liquid chromatography-mass spectrometry (LC-MS) and CE-MS, alternatively on the same mass spectrometer. The two separation techniques can be switched within minutes. To obtain a stable ion spray and avoid electrical problems, the CE-power supply is used to produce the potential for the CE separation and the ESI sprayer tip simultaneously. The necessary sheath liquid is delivered by a pump which floats on the ion sprayer potential of the mass spectrometer, avoiding any current flow towards ground. The sole parameter which has to be adjusted to adapt to different CE conditions is a variable resistor. Analytical applications such as peptide mixture analysis and drug screening are presented.
\end{abstract}

Keywords: Amphetamines · CE-MS · Interface · Peptides · Sheath flow

${ }^{*}$ Correspondence: P. Bernet ${ }^{\mathrm{a}}$

c/o Dr. M. Schär

Tel.: +4134426 4302

Fax: +41344264391

E-Mail: martin.schaer@isburg.ch

aBern University of Applied Sciences

School of Engineering and Information Technology

Division of Chemistry

Pestalozzistrasse 20

$\mathrm{CH}-3400$ Burgdorf

${ }^{\mathrm{b}}$ ReseaChem $\mathrm{GmbH}$

Pestalozzistrasse 16

$\mathrm{CH}-3400$ Burgdorf

\section{Introduction}

Capillary electrophoresis (CE) has become an essential tool in analytical chemistry. Its ability to separate ions as well as neutral compounds in small volumes with high theoretical plate numbers is indispensable for the analysis of complex mixtures. Theoretical plate counts from $10^{4}$ up to $10^{6}$ can be achieved.

The soft ionisation methods electrospray ionisation mass spectrometry (ESI-MS) and matrix-assisted laser desorption ionisation mass spectrometry (MALDI-MS) have been used routinely as bioanalytical tools in connection with powerful separation techniques. ESI-MS is especially well suited as an online detector for separation techniques such as liquid chromatography (HPLC) and CE. This is due to the high sensitivity, high selectivity and the possibility to obtain sequence information of biopolymers or structural details of smaller analyte molecules through MS/MS-methods.

A prerequisite for both the ESI-MS and $\mathrm{CE}$ methods is that the analytes have to be 